\title{
$\mathrm{SC}$

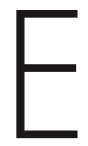

OPEN Data Descriptor: ROCK signalling \section{induced gene expression changes in mouse pancreatic ductal adenocarcinoma cells}

Received: 15 August 2016 Accepted: 26 September 2016 Published: 08 November 2016
Nicola Rath ${ }^{1}$, Gabriela Kalna ${ }^{1}$, William Clark $^{1}$ \& Michael F. Olson ${ }^{1,2}$

The RhoA and RhoC GTPases act via the ROCK1 and ROCK2 kinases to promote actomyosin contraction, resulting in directly induced changes in cytoskeleton structures and altered gene transcription via several possible indirect routes. Elevated activation of the Rho/ROCK pathway has been reported in several diseases and pathological conditions, including disorders of the central nervous system, cardiovascular dysfunctions and cancer. To determine how increased ROCK signalling affected gene expression in pancreatic ductal adenocarcinoma (PDAC) cells, we transduced mouse PDAC cell lines with retroviral constructs encoding fusion proteins that enable conditional activation of ROCK1 or ROCK2, and subsequently performed RNA sequencing (RNA-Seq) using the Illumina NextSeq 500 platform. We describe how gene expression datasets were generated and validated by comparing data obtained by RNA-Seq with RT-qPCR results. Activation of ROCK1 or ROCK2 signalling induced significant changes in gene expression that could be used to determine how actomyosin contractility influences gene transcription in pancreatic cancer.

\begin{tabular}{|l|l|}
\hline Design Type & $\begin{array}{l}\text { compound treatment design @ in vitro design } 0 \text { transcription profiling by high } \\
\text { throughput sequencing design } \bullet \text { genetic modification design }\end{array}$ \\
\hline Measurement Type(s) & transcription profiling assay \\
\hline Technology Type(s) & RNA sequencing \\
\hline Factor Type(s) & transgene - synthetic chemical compound - Replicate \\
\hline Sample Characteristic(s) & Mus musculus - pancreatic ductal adenocarcinoma cell \\
\hline
\end{tabular}

${ }^{1}$ Cancer Research UK Beatson Institute, Garscube Estate, Switchback Road, Glasgow G61 1BD, UK. ${ }^{2}$ Institute of Cancer Sciences, University of Glasgow, Glasgow G12 800, UK. Correspondence and requests for materials should be addressed to M.F.O. (email: m.olson@beatson.gla.ac.uk). 


\section{Background \& Summary}

ROCK1 and ROCK2 are serine/threonine kinases that play key roles in actomyosin cytoskeleton dynamics ${ }^{1}$. Acting in response to activation of their upstream regulators RhoA and RhoC GTPases, their regulation of cytoskeletal architecture gives them central influence on cell morphology, adhesion and motility ${ }^{2}$. ROCK proteins have been discovered to have important roles in differentiation, proliferation and apoptosis/survival in various cell types ${ }^{3}$. Given their roles in regulating cell motility, there has been considerable interest in developing ROCK inhibitors as cancer therapeutics to target invasion and metastasis ${ }^{4,5}$. It has more recently become appreciated that Rho-mediated actomyosin dynamics also impact gene transcription ${ }^{6}$. Despite many years of research, the effect of ROCK kinases in normal cell function or in disease states on the regulation of gene expression has not been well characterized.

Pancreatic cancer is the fourth leading cause of cancer-related deaths in the western world ${ }^{7,8}$. The most common form is pancreatic ductal adenocarcinoma (PDAC), with the majority of patients carrying activating KRAS oncogene mutations and mutation or deletion of the TP53 tumour suppressor?. Furthermore, exome sequencing of pancreatic cancer genomes revealed that $\sim 15 \%$ of pancreatic cancer patients carry ROCK 1 gene amplifications ${ }^{10}$. Additional genetic alterations that reduce TGF $\beta$ signalling, for example through loss of SMAD4 expression, result in elevated ROCK1-dependent cell contractility that promotes PDAC progression ${ }^{11,12}$. As a result, there are clear indications that ROCK signalling contributes to PDAC growth and progression, likely as an ancillary factor, through mechanisms that remain to be determined.

To investigate the contribution elevated ROCK signalling has on pancreatic cancer progression, we used pancreatic cancer cells previously isolated from a mouse PDAC tumour driven by $\mathrm{Kras}^{\mathrm{G} 12 \mathrm{D}} / \mathrm{p} 53^{+/-}$ (ref. 13) and retrovirally transduced constructs encoding ROCK1:ER, ROCK2:ER or GFP:ER fusion proteins (Fig. 1a $)^{14}$. The stable fusion protein expressing cell lines allow for conditional activation of ROCK kinase signalling by the addition of estrogen analogues including 4-hydroxytamoxifen (4HT) (Fig. 1b), which induces actomyosin contraction through phosphorylation and inhibition of the myosin phosphatase targeting subunit (MYPT) 1 and direct phosphorylation of the myosin light chains (MLC) (Fig. 1c). This is aided by LIM kinase 1 and 2 (LIMK1/2) phosphorylation, which in turn phosphorylate and inactivate the actin-severing cofilin proteins leading to actin filament stabilisation (Fig. 1c). Western blotting showed expression of GFP:ER, ROCK1:ER or ROCK2:ER fusion proteins in PDAC cells, with ROCK1:ER or ROCK2:ER expression comparable to endogenous ROCK1 or ROCK2 (Fig. 1d). To demonstrate conditional ROCK:ER activation, immunoblotting revealed 4HT-induced activation of ROCK1:ER or ROCK2:ER leading to increased MLC and LIMK1/2 phosphorylation that could be reversed by the inclusion of the ROCK inhibitor Y27632 (Fig. 1d).
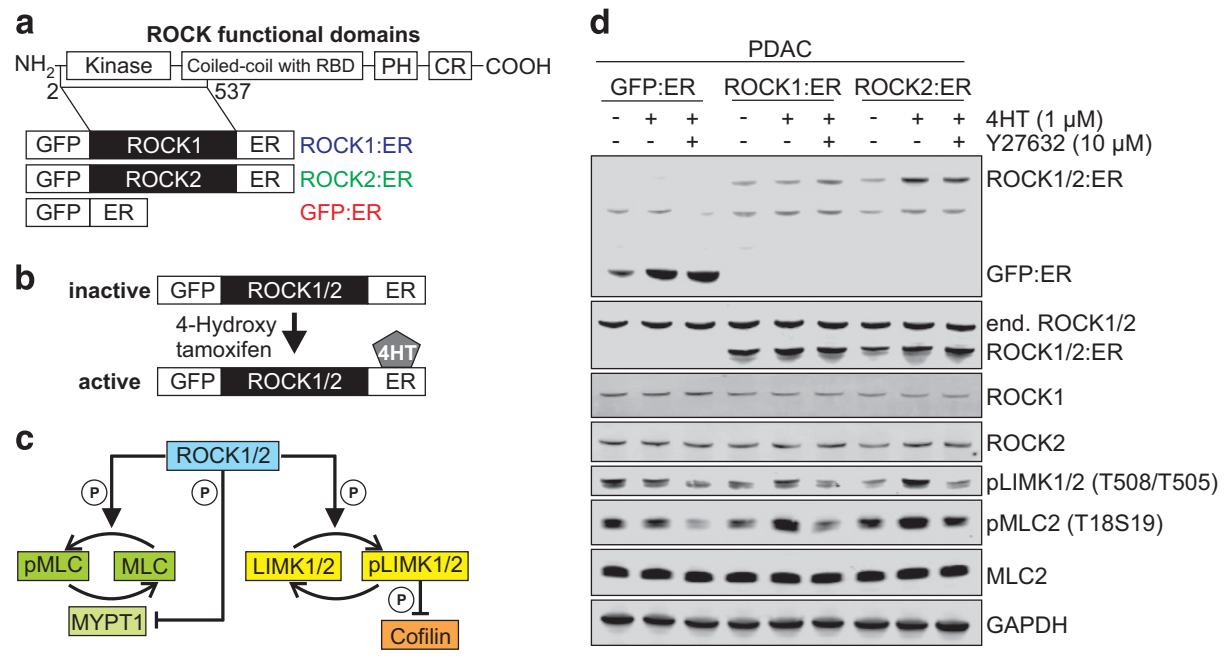

Figure 1. ROCK kinase activation in PDAC cells. (a) ROCK functional domains and ROCK kinase expression constructs (CR, cysteine-rich; ER, estrogen receptor hormone binding domain; GFP, green fluorescent protein; $\mathrm{PH}$, pleckstrin homology domain; RBD, Rho binding domain). (b) Conditional activation of ROCK kinase by 4-hydroxytamoxifen (4HT). (c) Overview of the ROCK signalling pathway. (d) PDAC cells expressing GFP:ER, ROCK1:ER or ROCK2:ER fusion proteins were treated with EtOH vehicle or $1 \mu \mathrm{M} 4 \mathrm{HT}$ in the presence or absence of $10 \mu \mathrm{M}$ Y 27632 ROCK inhibitor. Immunoblotting shows ER-fusion proteins, endogenous ROCK1 and ROCK2, and phosphorylation status of ROCK targets LIMK1/2 (T508/T505) and regulatory myosin light chain (MLC2; T18S19). Total MLC2 and glyceraldehyde-3-phosphate dehydrogenase (GAPDH) were blotted as loading controls. 


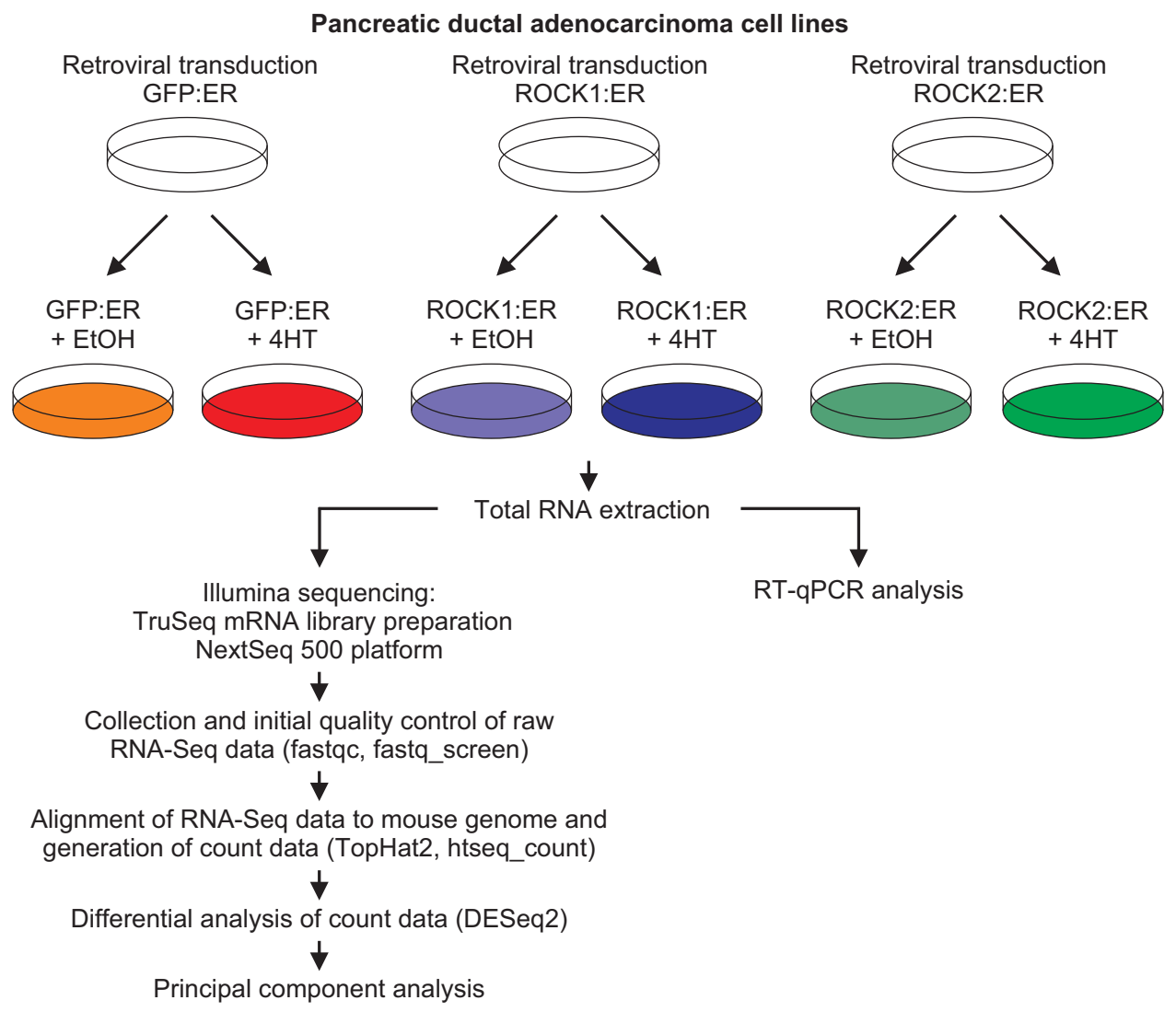

Figure 2. Overview and experimental design of the study.

To study the global effect of ROCK activation on gene expression in mouse PDAC cells, we sequenced polyA+ mRNAs of GFP:ER expressing cells treated with EtOH vehicle or 4HT, ROCK1:ER expressing cells treated with $4 \mathrm{HT}$ and ROCK2:ER cells treated with $4 \mathrm{HT}$ from 3 independent experiments (Fig. 2, Table 1). The study has been described at the NCBI Bioproject (Data Citation 1), with a description of the cells used at the NCBI BioSample (Data Citation 2). Primary data are available at the NCBI Sequence Read Archive (Data Citation 3).

\section{Methods}

\section{Cell culture}

A pancreatic ductal adenocarcinoma (PDAC) cell line was generated from the pancreatic tumour of a LSL-Kras ${ }^{\mathrm{G} 12 \mathrm{D} /+}$; LSL-p $53^{\mathrm{I} /+} ;$ Pdx1-Cre mouse ${ }^{13}$. Cells were maintained in High glucose DMEM (Gibco 21969-035) containing 10\% fetal bovine serum (FBS; Gibco), $2 \mathrm{mmoll}^{-1} \mathrm{~L}_{\text {-glutamine (Gibco), and }}$ penicillin-streptomycin (Gibco).

\section{Generation of stable cell lines}

Retroviral infection was used to generate stable PDAC cell lines expressing conditionally active human ROCK1 (ROCK1:ER), human ROCK2 (ROCK2:ER) or GFP control (GFP:ER). The retroviral pBABE puro constructs have been described previously ${ }^{14,15}$. Stable PDAC cell pools were selected using $2.5 \mu \mathrm{g} \mathrm{ml}^{-1}$ Puromycin using standard procedures.

\section{Western blot analysis}

$5 \times 10^{5}$ cells were seeded into 6-well plates in DMEM and allowed to settle and grow overnight. Next day, cells were starved in serum-free DMEM for $9 \mathrm{~h}$, followed by serum-free DMEM with EtOH vehicle or $1 \mu \mathrm{M} 4 \mathrm{HT}$ (Sigma H7904) in the presence or absence of $10 \mu \mathrm{M}$ Y27632 (Tocris 1254). After $16 \mathrm{~h}$ of treatment, whole cell lysates were prepared in cell lysis buffer (1\% SDS, $50 \mathrm{mM}$ Tris pH 7.5) and protein concentration determined by Bicinchoninic assay (Sigma). Standard protocols were used for Western blotting. Signals were detected by infrared imaging (Li-Cor Odyssey). Antibodies used: ROCK1 (BD-611136), ROCK2 (BD-610623), ROCK1/2 (Millipore 07-1458), phospho-Limk1/2 (Cell Signalling 3841), phospho-MLC2 (Cell Signalling 3674), MLC2 (Cell Signalling 3672), GFP (Abcam ab6556), GAPDH (Millipore MAB374), Alexa-Fluor 680 (life technologies) and DyLight 800 (Thermo Fisher Scientific). 


\begin{tabular}{|c|c|c|c|c|c|}
\hline Experiment & Fusion protein & Treatment & Data File ID & Total sequence reads & SRA accession \\
\hline 1 & GFP:ER & Ethanol (vehicle) & 1-EX1-GFP-EtOH & $3.8 \mathrm{E}+07$ & SRR4015461 \\
\hline 1 & GFP:ER & $4 \mathrm{HT}(1 \mu \mathrm{M})$ & 2-EX1-GFP-4HT & $6.4 \mathrm{E}+07$ & SRR4015530 \\
\hline 1 & ROCK1:ER & $4 \mathrm{HT}(1 \mu \mathrm{M})$ & 3-EX1-ROCK1-4HT & $5.8 \mathrm{E}+07$ & SRR4015566 \\
\hline 1 & ROCK2:ER & $4 \mathrm{HT}(1 \mu \mathrm{M})$ & 4-EX1-ROCK2-4HT & $6.2 \mathrm{E}+07$ & SRR4015572 \\
\hline 2 & GFP:ER & Ethanol (vehicle) & 5-EX2-GFP-EtOH & $6.8 \mathrm{E}+07$ & SRR4014833 \\
\hline 2 & GFP:ER & $4 \mathrm{HT}(1 \mu \mathrm{M})$ & 6-EX2-GFP-4HT & $6.3 \mathrm{E}+07$ & SRR4015468 \\
\hline 2 & ROCK1:ER & $4 \mathrm{HT}(1 \mu \mathrm{M})$ & 7-EX2-ROCK1-4HT & $5.7 \mathrm{E}+07$ & SRR4015485 \\
\hline 2 & ROCK2:ER & $4 \mathrm{HT}(1 \mu \mathrm{M})$ & 8-EX2-ROCK2-4HT & $7 \mathrm{E}+07$ & SRR4015492 \\
\hline 3 & GFP:ER & Ethanol (vehicle) & 9-EX3-GFP-EtOH & $6.1 \mathrm{E}+07$ & SRR4015510 \\
\hline 3 & GFP:ER & $4 \mathrm{HT}(1 \mu \mathrm{M})$ & 10-EX3-GFP-4HT & $6.3 \mathrm{E}+07$ & SRR4015519 \\
\hline 3 & ROCK1:ER & $4 \mathrm{HT}(1 \mu \mathrm{M})$ & 11-EX3-ROCK1-4HT & $6.1 \mathrm{E}+07$ & SRR4015548 \\
\hline 3 & ROCK2:ER & $4 \mathrm{HT}(1 \mu \mathrm{M})$ & 12-EX3-ROCK2-4HT & $6.3 \mathrm{E}+07$ & SRR4015560 \\
\hline
\end{tabular}

Table 1. Description and names of data files deposited with SRA.

\section{RNA isolation}

$1 \times 10^{6}$ cells were seeded into 6-well plates in DMEM and allowed to settle and grow overnight. Next day, cells were washed three times with serum-free DMEM, then serum-free DMEM with EtOH vehicle or $1 \mu \mathrm{M} 4 \mathrm{HT}$ (Sigma H7904) was added to the cells for $24 \mathrm{~h}$. Cells were harvested with Trypsin and total RNA was extracted using the RNAeasy kit (Qiagen) according to manufacturer's instructions. RNA was quantified using the Nanodrop spectrophotometer (Nanodrop Tech). The Agilent RNA ScreenTape assay and the Agilent 2200 TapeStation system were used to determine the RNA integrity number equivalent (RINe).

\section{RNA sequencing}

Total RNA was used to generate an oligo dT-enriched library with the Illumina TruSeq RNA Library Preparation kit v2.0. RNA fragmentation yielded fragments for use in preparation of the DNA library ranging from 120 to 210 nucleotides with a median of 155 nucleotides. Quality and quantity of the DNA library was assessed using the Agilent 2100 Bioanalyzer and the Qubit (Thermo Fisher Scientific), respectively. The library was run on the Illumina NextSeq 500 platform using the High Output 75 cycles kit $(2 \times 36$ cycles, paired-end reads, single index).

\section{RNA sequence analysis}

Quality control checks of raw RNA-Seq data files were done with fastqc (http://www.bioinformatics. babraham.ac.uk/projects/fastqc/) and fastq_screen (http://www.bioinformatics.babraham.ac.uk/projects/ fastq_screen/). RNA-Seq reads were aligned to the mouse genome (GRCm38.75) using TopHat2.0.10 (ref. 16) and BAM files were further processed with HTseq0.5.4p3 (http://www.huber.embl.de/users/anders/ HTSeq/doc/count.html) using standard configurations. The percentage of aligned reads was $88.6 \% \pm 2.0 \%$ (mean \pm s.d.). Differential analysis of count data was performed by the DESeq2 package (DESeq2) ${ }^{17}$. Regularized log transformation was used to transform the DESeq2 data for principal component analysis.

\section{Quantitative PCR}

Total RNA was used to synthesize complementary DNA with the QuantiTect Reverse Transcription Kit (Qiagen). Quantitative polymerase chain reactions (qPCR) were set up with the DyNAmo HS SYBR Green qPCR Kit (Thermo Fisher Scientific) and primers for Ptgs2, Tff3, Tnc, Cd44, Cyr61 or Gapdh (Quantitect Primer Assay, Qiagen). Reactions were run and analysed using the 7500 Fast Real-Time PCR System (Applied Biosystems).

\section{Data Records}

Unprocessed RNA sequencing reads have been deposited as fastq files at the National Center for Biotechnology Information (NCBI) Sequence Reads Archive (SRA) with the reference SRP081135. In addition, a project overview has been submitted as the BioProject reference PRJNA327913 (http:// www.ncbi.nlm.nih.gov/bioproject/) (Data Citation 1) with a description of the BioSample reference SAMN05361890 (http://www.ncbi.nlm.nih.gov/biosample/) (Data Citation 2).

The fastq files correspond to three independent experimental replicates (Experiment numbers 1-3) for the PDAC expressing GFP:ER cells treated with EtOH vehicle control or 4HT, or for the ROCK1:ER or ROCK2:ER expressing cells treated with $4 \mathrm{HT}$ as indicated in Table 1. Forward (R1) and reverse (R2) reads have been combined, with SRA accession numbers for the combined sequencing results also indicated in Table 1 (Data Citation 3). Please also see the associated Metadata Record. 


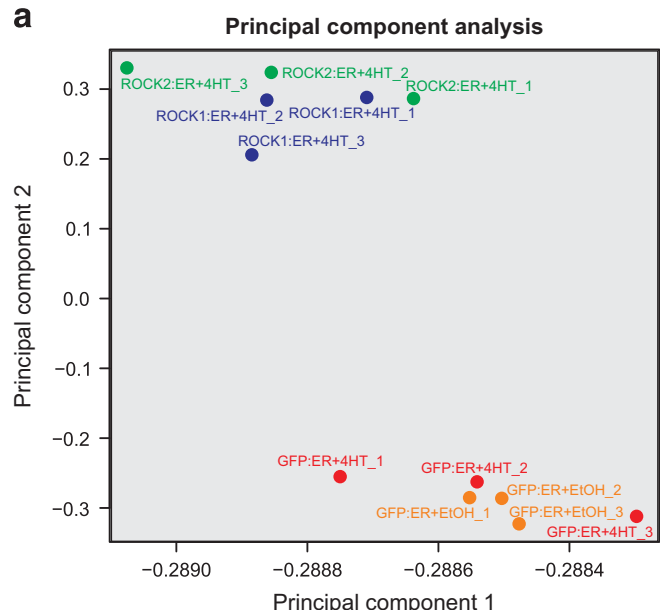

b

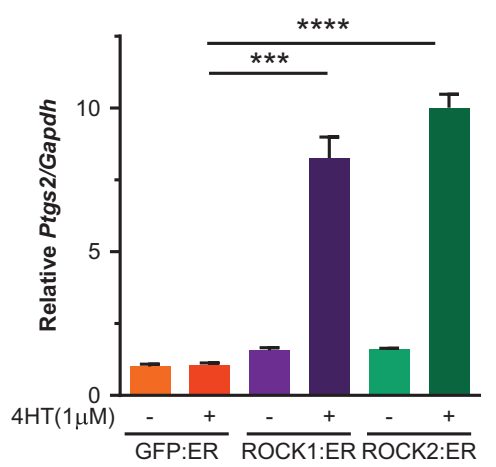

d

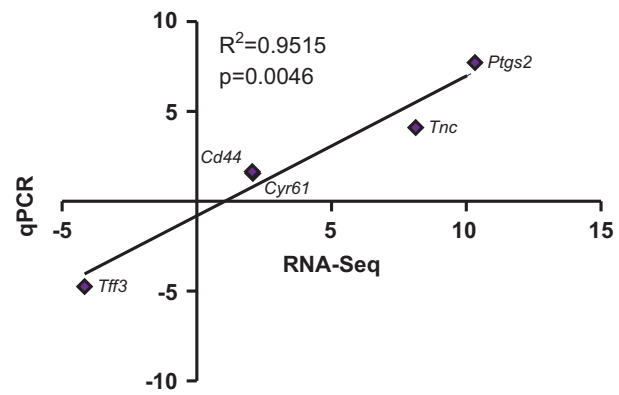

C

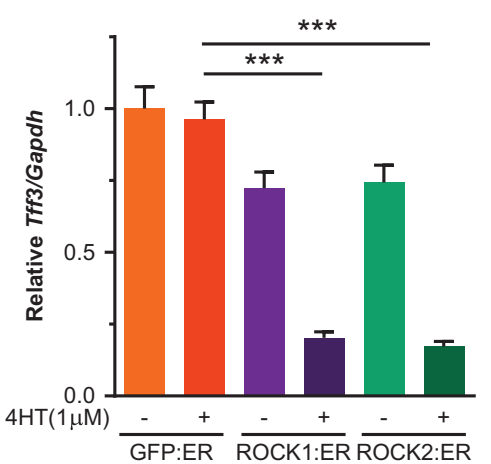

e ROCK2:ER+4HT vs GFP:ER+4HT (Fold change)

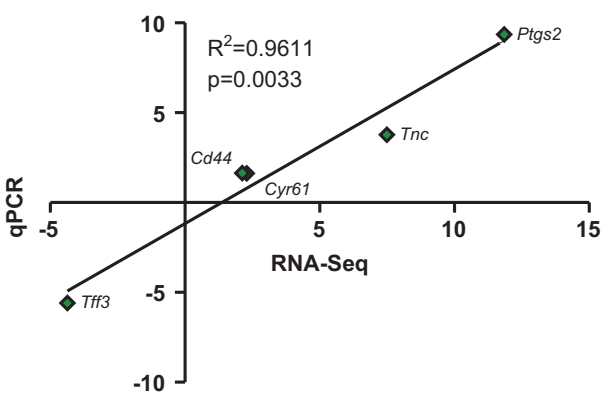

Figure 3. Quality control of RNA-Seq data. (a) Principal component plot of RNA-Seq (DESeq2) data indicating the clustering together of GFP:ER samples treated with vehicle control or 4HT, and ROCK1:ER plus ROCK2:ER samples treated with 4HT. (b) PDAC cells expressing GFP:ER, ROCK1:ER or (c) ROCK2:ER were treated with EtOH vehicle or $1 \mu \mathrm{M} 4 \mathrm{HT}$. Ptgs2 and Tff3 mRNA levels relative to Gapdh housekeeping gene were determined by qPCR. Means \pm s.e.m., unpaired $t$-test $(n=3)$. ${ }^{\star \star \star} P<0.001$. (d) Linear regression plots of fold change for GFP:ER+4HT versus ROCK1:ER+4HT or (e) GFP:ER+4HT versus ROCK2:ER+4HT obtained by RNA-Seq (DESeq2 data) compared to RT-qPCR (relative to Gapdh). RNA transcripts up-regulated by ROCK activation: Ptgs2, Tnc, Cd44 and Cyr61. RNA transcript down-regulated by ROCK activation: Tff3. $\mathrm{R}^{2}$ of Goodness of Fit with $\mathrm{p}$ value $(n=3)$.

\section{Technical Validation}

\section{Quality control of RNA-Seq data}

RNA quality was confirmed using the Agilent RNA ScreenTape assay and the Agilent 2200 TapeStation system, which revealed RNA integrity number equivalent $\left(\mathrm{RIN}^{\mathrm{e}}\right)$ values of 10 for all samples. Following RNA-seq, principal component analysis indicated that GFP:ER samples that had been treated with EtOH vehicle or $4 \mathrm{HT}$ clustered together while ROCK1:ER and ROCK2:ER samples treated with 4HT clustered together separate from the GFP:ER grouping (Fig. 3a), consistent with the conditional activation of ROCK catalytic activity observed by western blotting (Fig. 1d). Quantitative reverse transcription PCR 
(RT-qPCR) analyses validated differences in gene expression upon ROCK1:ER or ROCK2:ER activation identified by RNA seq, including increased Protaglandin-endoperoxidase 2 (Ptgs2) RNA (Fig. 3b) and decreased Trefoil factor 3 (Tff3) RNA (Figure 3c). We further validated the changes in gene expression identified by RNA seq by comparing fold changes in sequence reads with fold changes determined by RT-qPCR for Ptgs2, Tff3, Cyr61, Tnc and Cd44 between 4HT treated GFP:ER versus ROCK1:ER (Fig. 3d) and GFP:ER versus ROCK2:ER (Fig. 3e) conditions. In both cases, the fold-changes determined by either method fell on a single fitted straight line with $\mathrm{R}^{2}>0.95$ and $P<0.05$ (Fig. 3d,e). Although there was good agreement between RNA seq and RT-qPCR for this limited gene set, it is possible that the analytical methods used may have underestimated gene expression levels ${ }^{18}$.

\section{References}

1. Julian, L. \& Olson, M. F. Rho-associated coiled-coil containing kinases (ROCK). Small GTPases 5, e29846 (2014).

2. Thumkeo, D., Watanabe, S. \& Narumiya, S. Physiological roles of Rho and Rho effectors in mammals. Eur. J. Cell Biol. 92, 303-315 (2013)

3. Loirand, G. Rho Kinases in Health and Disease: From Basic Science to Translational Research. Pharmacol. Rev. 67, 1074-1095 (2015).

4. Rath, N. \& Olson, M. F. Rho-associated kinases in tumorigenesis: re-considering ROCK inhibition for cancer therapy. EMBO Rep. 13, 900-908 (2012).

5. Chin, V. T. et al. Rho-associated kinase signalling and the cancer microenvironment: novel biological implications and therapeutic opportunities. Expert. Rev. Mol. Med. 17, e17 (2015).

6. Rajakylä, E. K. \& Vartiainen, M. K. Rho, nuclear actin, and actin-binding proteins in the regulation of transcription and gene expression. Small GTPases 5, e27539 (2014).

7. Siegel, R. L., Miller, K. D. \& Jemal, A. Cancer statistics, 2015. CA. Cancer J. Clin. 65, 5-29 (2015).

8. Weledji, E. P., Enoworock, G., Mokake, M. \& Sinju, M. How Grim is Pancreatic Cancer? Oncol. Rev. 10, 294 (2016).

9. Polireddy, K. \& Chen, Q. Cancer of the Pancreas: Molecular Pathways and Current Advancement in Treatment. J. Cancer 7, 1497-1514 (2016).

10. Biankin, A. V. et al. Pancreatic cancer genomes reveal aberrations in axon guidance pathway genes. Nature 491, 399-405 (2012).

11. Laklai, H. et al. Genotype tunes pancreatic ductal adenocarcinoma tissue tension to induce matricellular fibrosis and tumor progression. Nat. Med. 22, 497-505 (2016)

12. Rath, N. \& Olson, M. F. Regulation of pancreatic cancer aggressiveness by stromal stiffening. Nat. Med. 22, 462-463 (2016).

13. Morton, J. P. et al. Mutant p53 drives metastasis and overcomes growth arrest/senescence in pancreatic cancer. Proc. Natl Acad Sci. USA 107, 246-251, d (2010).

14. Croft, D. R. \& Olson, M. F. Conditional Regulation of a ROCK-Estrogen Receptor Fusion Protein. Methods Enzymol. 406, 541-553 (2006)

15. Croft, D. et al. Actin-myosin-based contraction is responsible for apoptotic nuclear disintegration. J. Cell Biol. 168, 245-255 (2005).

16. Kim, D. et al. TopHat2: accurate alignment of transcriptomes in the presence of insertions, deletions and gene fusions. Genome Biol. 14, R36 (2013).

17. Love, M. I., Huber, W. \& Anders, S. Moderated estimation of fold change and dispersion for RNA-seq data with DESeq2. Genome Biol. 15, 550 (2014).

18. Robert, C. \& Watson, M. Errors in RNA-Seq quantification affect genes of relevance to human disease. Genome biology 16, $1-16(2015)$.

\section{Data Citations}

1. NCBI BioProject PRJNA327913 (2016).

2. NCBI BioSample SAMN05361890 (2016).

3. NCBI Sequence Reads Archive SRP081135 (2016).

\section{Acknowledgements}

Funding for this project was from Cancer Research U.K. (A18276).

\section{Author Contributions}

Experiments were designed by N.R. and M.F.O., and performed by N.R. Enrichment of total RNA for poly $^{+}$fraction, library construction and sample analysis on the Illumina NextSeq 500 platform were done by W.C. Sequence assembly and statistical analysis were performed by G.K. Figures were prepared by N.R. Manuscript was written by N.R. and M.F.O.

\section{Additional Information}

Competing financial interests: The authors declare no competing financial interests.

How to cite: Rath, N. et al. ROCK signalling induced gene expression changes in mouse pancreatic ductal adenocarcinoma cells. Sci. Data 3:160101 doi: 10.1038/sdata.2016.101 (2016).

Publisher's note: Springer Nature remains neutral with regard to jurisdictional claims in published maps and institutional affiliations.

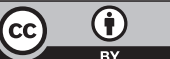

This work is licensed under a Creative Commons Attribution 4.0 International License. The images or other third party material in this article are included in the article's Creative Commons license, unless indicated otherwise in the credit line; if the material is not included under the Creative Commons license, users will need to obtain permission from the license holder to reproduce the material. To view a copy of this license, visit http://creativecommons.org/licenses/by/4.0

Metadata associated with this Data Descriptor is available at http://www.nature.com/sdata/ and is released under the CCO waiver to maximize reuse.

(c) The Author(s) 2016 